\title{
AUTODETERMINACIÓN EN EL ÁMBITO EDUCATIVO EN ADOLESCENTES CON TRASTORNO DEL ESPECTRO AUTISTA
}

\section{Self-Determination in the Educational Environment in Adolescents with Autism Spectrum Disorder}

\author{
Soraya Sánchez CASAdo \\ Universidad de Salamanca \\ ssanchezc@usal.es \\ Elena MARTÍn-PASTOR \\ Universidad de Salamanca \\ Isabel Calvo Álvarez \\ Universidad de Salamanca
}

Recepción: 16 de abril de 2020

Aceptación: 4 de diciembre de 2020

Resumen: La autodeterminación constituye una de las dimensiones clave en la definición de la calidad de vida de las personas. Este trabajo se centra en analizar dicha dimensión en adolescentes con Trastorno del Espectro Autista (TEA) en el contexto escolar, como uno de los espacios de mayor relevancia y desarrollo personal de esta etapa vital. Concretamente se presenta una revisión sistemática con la finalidad de conocer el estado de autodeterminación que presentan los adolescentes con autismo en el ámbito educativo, variables que más influyen en su consecución e intervenciones desarrolladas para un mayor logro de conducta autodeterminada. De una búsqueda inicial de 414 trabajos, únicamente siete cumplieron los criterios definidos. Los principales resultados obtenidos se concretan en niveles bajos de autodeterminación para este colectivo, donde variables como el nivel de discapacidad, el género, la edad, etc., se convierten en las principales barreras. A ello se suma la escasez de programas de mejora de la conducta autodeterminada. Lo anterior desvela la necesidad de que la autodeterminación adquiera mayor protagonismo en el diseño de los programas educativos.

Palabras clave: autodeterminación; Trastorno del Espectro Autista; adolescentes; ámbito educativo. 
АвsтRACт: Self-determination constitutes a key dimension in the definition of people's quality of life. This work focuses on analysing this dimension in adolescents with Autism Spectrum Disorder (ASD) in the school setting, since it is considered one of the most relevant places for personal development at this life stage. Specifically, a systematic review is presented with the aim of obtaining more significant information on the level of self-determination among adolescents with autism within the educational scope, the variables that influence the most in their achievement and the interventions developed for a greater accomplishment of self-determined behaviour. From an initial research of 414 studies, only seven met the defined criteria. Main results achieved reflect low levels of self-determination for this group, where variables such as the level of disability, gender, age, etc., become the main barriers. Added to this is the shortage of self-determination improvement programmes. All this reflects the need for self-determination to gain greater visibility throughout the design of educational programmes.

KeYwords: Self-determination; Autism Spectrum Disorders; teenagers; educational environment.

\title{
1. Introducción
}

\begin{abstract}
egún la quinta edición del Manual Diagnóstico y Estadístico de Trastornos Mentales (DSM-5) (American Psychiatry Association, 2018), el Trastorno del Espectro Autista (TEA) se encuentra catalogado dentro de los trastornos del neurodesarrollo. Las personas con este trastorno se caracterizan por manifestar continuas deficiencias en lo que tiene que ver con la comunicación social y las relaciones interpersonales en diferentes contextos. Presentan ciertas dificultades en la interdependencia socioemocional; en las habilidades de comunicación no verbales y verbales usadas en la interacción con otras personas, y dificultades en el hecho de desarrollar, mantener y comprender las relaciones sociales. También, muestran patrones restrictivos y repetitivos en su conducta comportamental, en sus intereses o actividades, manifestándose en dos o más de los síntomas siguientes:
\end{abstract}

Movimientos, utilización de objetos o habla estereotipados o repetitivos, 2. Insistencia en la monotonía, excesiva inflexibilidad de rutinas, 3. Intereses muy restringidos y fijos que son anormales en cuanto a su intensidad o foco de interés y, 4. Hiper- o hiporreactividad a los estímulos sensoriales o interés inhabitual por aspectos sensoriales del entorno. (American Psychiatry Association, 2018, p. 29)

Estos síntomas se manifiestan en las primeras fases del proceso de desarrollo causando un deterioro significativo en el área social, laboral u otras que resultan substanciales para un habitual funcionamiento humano. Asimismo, hay que tener en cuenta que estas alteraciones no vienen mejor identificadas por presentar discapacidad intelectual (trastorno del desarrollo intelectual) o un retraso global del desarrollo. León (2008) afirma que el déficit cognitivo global se observa en la mayoría de las personas 
con TEA, mostrando, también, graves problemas en las relaciones sociales y en la comunicación, al igual que problemas conductuales, lo que conlleva una importante afectación de su autonomía. Del mismo modo, la American Psychiatry Association (2018) informa que la gravedad del trastorno se basará en el deterioro de la comunicación social y en los modelos de comportamientos repetitivos y restringidos. Esta gravedad se encontrará registrada conforme al grado de ayuda que cada persona necesite (Tabla 1).

\begin{tabular}{|l|l|l|}
\hline \multicolumn{2}{|c|}{ TABLA 1. Niveles de gravedad del trastorno del espectro autista } \\
\hline Nivel de gravedad & Comunicación social & $\begin{array}{l}\text { Comportamientos restringidos } \\
\text { y repetitivos }\end{array}$ \\
\hline $\begin{array}{l}\text { Grado 3: "Necesita } \\
\text { ayuda muy notable" }\end{array}$ & $\begin{array}{l}\text { Graves deficiencias en habilidades } \\
\text { comunicativas verbales y no verba- } \\
\text { les que ocasionan severas alteracio- } \\
\text { nes en el funcionamiento humano, } \\
\text { con un limitado inicio de relaciones } \\
\text { interpersonales y escasa apertura } \\
\text { social. }\end{array}$ & $\begin{array}{l}\text { Comportamientos inflexibles, graves } \\
\text { dificultades para enfrentarse a cam- } \\
\text { bios o diversos comportamientos re- } \\
\text { petivos y restringidos que producen } \\
\text { notables interferencias en el funciona- } \\
\text { miento humano en todos los contex- } \\
\text { tos. Al cambiar la conducta o el foco } \\
\text { de interés presentan problemas de an- } \\
\text { siedad o dificultades intensas. }\end{array}$ \\
\hline $\begin{array}{l}\text { Grado 2: "Necesita } \\
\text { ayuda notable" }\end{array}$ & $\begin{array}{l}\text { Notables deficiencias en habilidades } \\
\text { comunicativas verbales y no verba- } \\
\text { les, con aparentes problemas socia- } \\
\text { les aun disponiendo de apoyos y } \\
\text { limitado inicio de relaciones inter- } \\
\text { personales y escasa apertura social. }\end{array}$ & $\begin{array}{l}\text { Comportamientos inflexibles, dificul- } \\
\text { tades para enfrentarse a cambios o di- } \\
\text { versos comportamientos repetitivos y } \\
\text { restringidos (apareciendo con notable } \\
\text { frecuencia, pudiendo ser obvios para } \\
\text { un observador que no esté entrena- } \\
\text { do), produciendo interferencias en el } \\
\text { funcionamiento humano en diversos } \\
\text { ámbitos. Al cambiar la conducta o el } \\
\text { foco de interés presentan ansiedad o } \\
\text { dificultad. }\end{array}$ \\
\hline $\begin{array}{l}\text { Grado 1: "Necesita } \\
\text { ayuda" }\end{array}$ & $\begin{array}{l}\text { Sin ayuda, los problemas de comu- } \\
\text { nicación social producen impor- } \\
\text { tantes alteraciones. Problemas para } \\
\text { iniciar las relaciones interpersona- } \\
\text { les, presentando atípicas o fallidas } \\
\text { respuestas a la apertura social. Pare- } \\
\text { ce que su interés por la interacción } \\
\text { social es reducido. }\end{array}$ & $\begin{array}{l}\text { Los comportamientos inflexibles oca- } \\
\text { sionan significativas interferencias en } \\
\text { el funcionamiento humano en uno } \\
\text { o más entornos. Su autonomía se ve } \\
\text { afectada por sus problemas de plani- } \\
\text { ficación y organización. }\end{array}$ \\
\hline
\end{tabular}

Fuente: Modificada de Bonilla y Chaskel (2016) según la American Psychiatric Association. Diagnostic and Statistical manual of mental disorders (DSM-5). 
Para Baña (2011), hablar de trastornos del espectro autista y generales del desarrollo es hablar de vidas, de personas con calidad de vida, entendida esta como una finalidad, pero también como una forma de vida que permite optimizar los procesos de aprendizaje y de desarrollo.

Puntualizar que la calidad de vida es entendida según Schalock y Verdugo (2007) como "un estado deseado de bienestar personal que: (a) es multidimensional; (b) tiene propiedades éticas -universales-y émicas -ligadas a la cultura- (c) tiene componentes objetivos y subjetivos; y (d) está influenciada por factores personales y ambientales" (p. 22).

A su vez, la calidad de vida de una persona se encuentra definida por las ocho dimensiones que la componen, siendo estas según Verdugo et al. (2013): bienestar emocional, relaciones interpersonales, bienestar material, desarrollo personal, bienestar físico, inclusión social, derechos y autodeterminación.

Esta última dimensión es en la que se centrará este trabajo. Wehmeyer (2005) señala que la autodeterminación de una persona se refiere a su capacidad para realizar acciones volitivas -con autonomía - que le permitan actuar como el principal agente causal de su vida y con ello avanzar hacia la consecución de los objetivos fijados. Asimismo, Arellano y Peralta (2013), siguiendo el modelo de Schalock y Verdugo, la entienden como la habilidad para poder decidir y llevar a cabo elecciones por uno mismo, teniendo como guía los objetivos, preferencias, elecciones, decisiones y autonomía propios.

Fernández et al. (2015) señalan que las personas con trastorno del espectro autista son uno de los grupos que presentan más problemas de autodeterminación dentro de la sociedad. El aprendizaje de la conducta autodeterminada se relaciona con el nivel de vida independiente, lo que nos posibilita tomar nuestras propias decisiones y, a su vez, ver las consecuencias de estas. Es por ello que Baña (2011) resalta la necesidad de dejar que las personas con TEA aprendan a tomar sus propias decisiones y actuar por sí mismas, aprendiendo las consecuencias de estas decisiones y acciones, de tal forma que irán siendo conscientes de la capacidad de la que disponen para dirigir su propia vida. Por este motivo, alega que la autodeterminación es imprescindible para disponer de una buena calidad de vida.

En el ámbito escolar, la autodeterminación no es solo un objetivo educativo, sino también un modo de aumentar la calidad de vida de los alumnos con TEA y un referente crucial para tomar decisiones curriculares (González y Peralta, 2006). De tal manera que, si el fin último de la educación es el de asegurar una independencia para la adecuada inserción social de su alumnado, este necesita aprender las estrategias necesarias para llegar a conseguirla. El desarrollo de la capacidad de autodeterminación debe estar incluido, por tanto, en el currículo obligatorio, pero no solo de manera puntual, sino de forma transversal y longitudinal en todas y cada una de las materias que lo configuran. La educación tiene que estar centrada por y para la persona en todas sus dimensiones, complementándola y ayudándola a llevar una vida plena en cada una de sus áreas (Tamarit, 2005).

La inclusión educativa se configura como el medio más efectivo para conseguir este reto, entendida como un proyecto de participación social y ciudadanía que demanda 
procesos de mejora y cambio que faciliten acogida y bienestar a todo el alumnado, pero, fundamentalmente, a los más vulnerables, aquellos con más posibilidad de fracaso, de quedar a los márgenes del sistema educativo y de que no se garanticen sus derechos (Arnaiz, 2019; Echeita 2017). No es por ello de extrañar que la inclusión se haya convertido en uno de los objetivos prioritarios para la Agenda 2030 del Desarrollo Sostenible a través del desarrollo de sistemas educativos que fomenten una educación inclusiva de calidad que promueva oportunidades de aprendizaje permanente para todos (UNESCO, 2017). Desde esa premisa de para todos, ya Wehmeyer en 2009, en su esfuerzo por dar un mayor protagonismo a la promoción de la autodeterminación desde el contexto escolar, señaló la importancia de eliminar los programas educativos basados en etiquetas y sustituirlos por el diseño de estrategias de apoyo generalizadas para toda el aula, y centradas en los puntos fuertes de los estudiantes.

Es por tanto fundamental que este modelo educativo guíe las políticas sociales y educativas, y esté presente en las aulas y centros educativos, para que no quede reducido a ideales y principios que no conllevan una transformación de la realidad educativa (Arnaiz, 2019).

Contemplando el marco normativo que regula y vertebra el funcionamiento del sistema educativo español nos encontramos con que la Ley Orgánica 8/2013, de 9 de diciembre, para la mejora de la calidad educativa, indica que los alumnos son el centro y la razón de ser del ámbito educativo. A su vez, hace hincapié en que "el aprendizaje en la escuela debe ir dirigido a formar personas autónomas, críticas, con pensamiento propio. Todos los alumnos y alumnas tienen un sueño, todas las personas jóvenes tienen talento. Nuestras personas y sus talentos son lo más valioso que tenemos como país” ( $B O E$, núm. 295, p. 3). Del mismo modo, explica que la reforma llevada a cabo tiene el propósito de evolucionar hacia un sistema que sea capaz de orientar a sus alumnos hacia trayectorias que sean lo más adecuadas a sus capacidades para que puedan lograr sus aspiraciones y puedan convertirse en caminos que faciliten la empleabilidad e inciten su espíritu emprendedor a través de la posibilidad, "para el alumnado y sus padres, madres o tutores legales, de elegir las mejores opciones de desarrollo personal y profesional” ( $B O E$, núm. 295, p. 3).

Sin embargo, a pesar de estas intenciones, al hacer una revisión del currículo para cada una de las etapas que componen el sistema educativo no se observan aspectos relacionados con la autodeterminación. Más bien se centra en la consecución de los objetivos de cada enseñanza y nivel educativo, manifestando más interés por los resultados académicos que por el desarrollo personal, mostrando, así, una carencia en aspectos de autodeterminación en el ámbito educativo, cuestión fundamental en el proceso de desarrollo y aprendizaje de cualquier estudiante (Shogren et al., 2006). De hecho, Yianni-Coudurier et al. (2008) alegan que los estudiantes con TEA tienen menores posibilidades de obtener recursos de apoyo específicos cuando se encuentran dentro de un contexto de educación general, lo que conlleva el hecho de que sus oportunidades se minimicen a la hora de desarrollar y realizar conductas que les dirijan a mejorar su autodeterminación. Es por ello que debería prestarse una mayor atención y disponer de más recursos para dotar a los docentes de modelos de intervención que hagan hincapié en la promoción de 
habilidades relacionadas con el comportamiento autodeterminado (MumbardóAdam et al., 2017). En la misma línea Shogren et al. (2015) señalan que, en el caso de adolescentes con discapacidad, "es una práctica óptima en la educación secundaria y en los servicios de transición” (p. 253). Esto es así porque la conducta autodeterminada hace posible que se lleve a cabo un desempeño académico, unos resultados de transición y de recreación más positivos, así como una mejor calidad de vida.

Debido a la importancia que la autodeterminación juega en el desarrollo integral de una persona, donde la edad escolar resulta un momento clave, este trabajo tiene la finalidad de conocer los estudios que se han llevado a cabo bajo el objetivo de analizar la conducta autodeterminada de los adolescentes con TEA, contribuyendo a definir la calidad de vida de estos escolares. Este objetivo general puede concretarse en cuatro específicos. Por un lado: (a) conocer los instrumentos de evaluación empleados para evaluar la autodeterminación en la población seleccionada, (b) analizar las diferentes variables que les pueden identificar como personas más autodeterminadas. Por otro lado, y a partir de los resultados que arrojen los estudios analizados: (c) conocer programas/intervenciones que pongan en marcha o mejoren los procesos de autodeterminación de los adolescentes con TEA en el ámbito educativo, (d) identificar las barreras que les dificultan el camino hacia la autodeterminación.

Para la consecución de estos objetivos, se ha realizado una revisión sistemática. En los siguientes apartados abordaremos las estrategias de búsqueda, el procedimiento y selección de artículos; posteriormente, expondremos los resultados más destacados.

\section{Metodología}

Se llevó a cabo una búsqueda de estudios sobre el tema objeto de investigación, utilizándose diferentes bases de datos: ERIC, MEDLINE, PsycINFO, PSICODOC, PubMed, Tripdatabase, Google Académico y Dialnet.

Se establecieron criterios de exclusión antes de iniciar la búsqueda: (1) rechazar aquellos artículos que no abordaran el tema de la autodeterminación de los adolescentes con TEA en el contexto escolar y (2) artículos que en su título, palabras claves o resumen no tuvieran los aspectos relevantes para llevar a cabo el análisis.

Para depurar la búsqueda se identificaron, además, una serie de criterios de inclusión: (1) los artículos deben abordar el tema de la autodeterminación; (2) los participantes deben ser adolescentes diagnosticados de Trastorno del Espectro Autista (TEA); (3) la edad de los participantes debe estar comprendida entre los 12 y los 21 años $^{1} ;$; (4) los participantes deben estar inmersos en el sistema educativo (colegios ordinarios o colegios de educación especial); (5) el año de publicación de los artículos debe ser entre 2013 y 2019, es decir, publicados en los últimos 5 años, de tal forma que tuviéramos una bibliografía lo más actualizada posible; (6) el contexto donde

Según el artículo 74 de la Ley Orgánica 2/2006, de 3 de mayo, de Educación, la escolarización de un alumno con necesidades educativas especiales podrá extenderse hasta los 21 años de edad en centros o unidades de educación especial ( $B O E$, núm. 106). 
se desarrollen los estudios puede ser nacional e internacional, y (7) el idioma de los artículos debe ser en inglés o en español.

Así, los términos de búsqueda utilizados fueron los siguientes:

- Self-determination AND autism spectrum disorders.

- Self-determination AND autism spectrum disorders NOT adults.

- Self-determination AND autism NOT adults.

- Self-determination AND ASD NOT adults.

Tras la búsqueda realizada en EBSCOhost, donde se incluyeron las bases de datos MEDLINE, ERIC, PsycINFO y PSICODOC, aplicando los anteriores criterios de búsqueda, se hallaron 93 artículos, y, después de analizar el título y las palabras claves, se seleccionaron 21 de ellos. En PubMed, se hallaron 32 artículos, de los cuales se seleccionaron 3, analizando el título y las palabras claves, como en las bases de datos anteriores. En Google Académico y Dialnet, se llevó a cabo el mismo proceso, encontrando, respectivamente, 11 y 4, seleccionando, finalmente, 10 y 1 . Por último, se procedió a realizar la búsqueda en Tripdatabase, donde aparecieron 274 artículos, pero ninguno de ellos cumplía el criterio del título y las palabras claves, por lo que se descartaron al no cumplir los criterios de inclusión establecidos.

De los 35 artículos seleccionados, se procedió a eliminar los artículos duplicados, obteniendo en este momento 28 artículos. A continuación, se revisó el resumen de cada uno de los artículos, quedándonos con una selección de 19. Asimismo, se procedió a leer el texto completo de los artículos seleccionados para comprobar si cumplían con los criterios de inclusión y exclusión que se especificaron con anterioridad.

Finalmente, 7 artículos cumplieron los criterios de inclusión establecidos para realizar este trabajo. A continuación, se presenta el diagrama de flujo (Figura 1) donde se recoge el proceso llevado a cabo. 


\section{FIGURA 1. Diagrama de flujo del proceso de búsqueda}
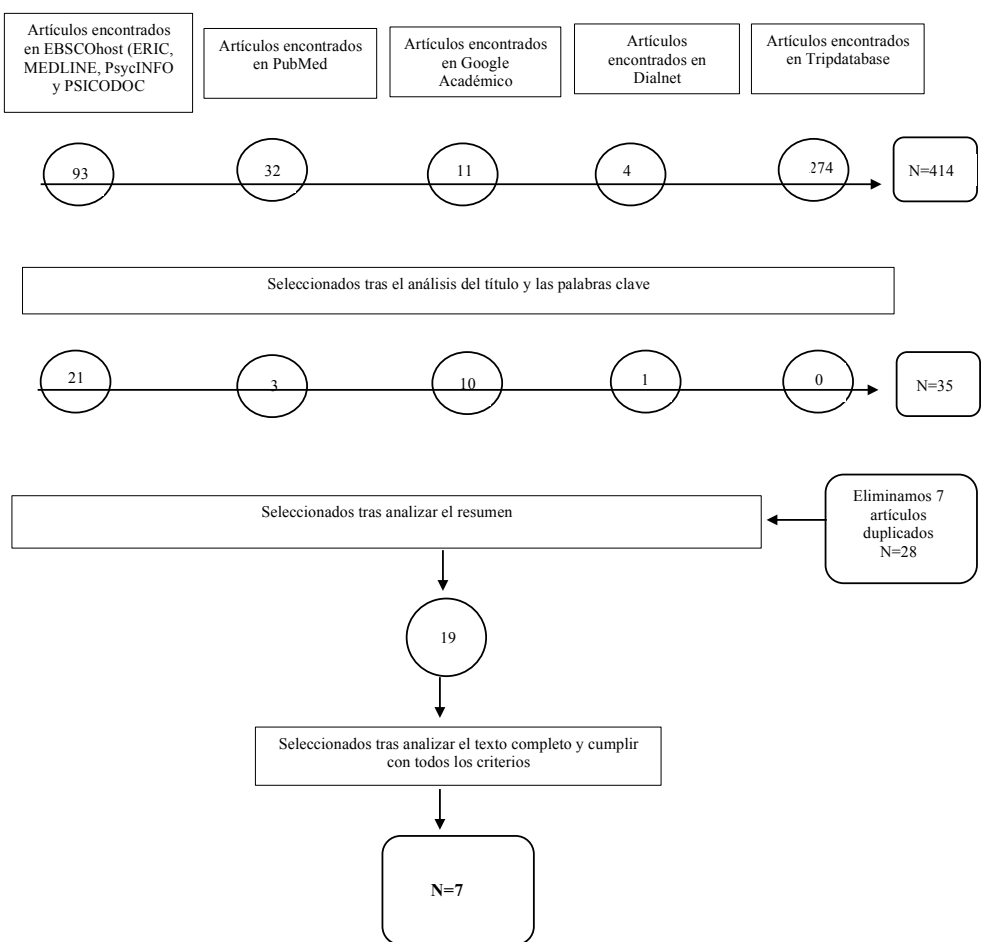

Fuente: Elaboración propia

\section{Resultados}

La búsqueda realizada en las diferentes bases de datos arrojó 414 artículos y, una vez aplicados los distintos criterios, se procedió a analizar 7 de ellos, aquellos que cumplían los criterios establecidos.

Los elementos seleccionados como más relevantes de cada uno de los artículos fueron título, autor/es, año de publicación, objetivos, población y muestra, el tipo de estudio, instrumentos de recogida de datos y las variables empleadas y, por último, los resultados y conclusiones más relevantes.

Cabe señalar que, a la hora de seleccionar los artículos, también se tuvieron en cuenta aquellos en los que los participantes fueran familiares y profesionales (de diferente ámbito: educativo, social, sanitario) que estaban en contacto con la población de interés, los adolescentes con trastorno del espectro autista. De modo que, exceptuando dos de los artículos, donde los informantes eran los propios estudiantes, el resto estuvo compuesto por personas del entorno de los propios escolares. 
Por otro lado, el objetivo de uno de los artículos, cuyos participantes son los propios estudiantes con TEA, no es arrojar resultados relacionados con su autodeterminación, sino analizar la fiabilidad y validez de dos escalas (Chou et al., 2015).

En relación a la edad de los adolescentes con autismo, o de los hijos o alumnos de padres y profesionales que participaron en las investigaciones analizadas, todos ellos eran adolescentes, la mayoría comprendidos en un rango de edad de 13 a 21 años, sin embargo, en tres de los artículos se ampliaba el rango de 3 a 21 años. En la Tabla 2, se recogen datos sobre la muestra total de los siete estudios seleccionados.

Centrándonos en los instrumentos utilizados, se observa bastante heterogeneidad. Todos ellos evalúan y exploran el estado de la autodeterminación de las personas con autismo, recogiendo los datos de forma cuantitativa, cualitativa o de ambas formas. Fernández et al. (2015) y García et al. (2017) coinciden en la utilización de la escala KidsLife, mientras que la Arc's Self-Determination Scale (SDS) fue el instrumento utilizado en los artículos de Chou et al. (2015) y Chou et al. (2016). Por otro lado, Carter et al. (2013a) y Carter et al. (2013b) realizaron su propia herramienta donde recogían información de forma cuantitativa y cualitativa, sirviéndose para la parte cuantitativa de la American Institutes for Research Self-Determination Scale (AIR). Puntualizar que este segundo trabajo (Carter et al., 2013b) constituye una continuación de la primera investigación, donde lo que cambia es la edad de la muestra. Por último, Timmons et al. (2017) utilizan una entrevista cualitativa diseñada por ellos.

\begin{tabular}{|l|c|c|c|}
\hline \multicolumn{4}{|c|}{ TABLA 2. Muestra total de los siete artículos seleccionados } \\
\hline & Padres y profesionales & $\begin{array}{c}\text { Hijos o alumnos } \\
\text { con TEA }\end{array}$ & Adolescentes con TEA \\
\hline Varones & $* 1$ & 349 & 146 \\
\hline Mujeres & $* 2$ & 68 & 29 \\
\hline Total & 797 & 434 & 175 \\
\hline
\end{tabular}

*1*2 No se dispone de información sobre el género de los padres y profesionales que participaron en los estudios seleccionados, ya que no se recogen estos datos en ninguno de los artículos. Fuente: Elaboración propia

Todos los instrumentos evalúan habilidades o elementos de la conducta autodeterminada: hacer elecciones, tomar decisiones, resolución de problemas, establecimiento y logro de metas, autodefensa y liderazgo, autogestión y autorregulación y autoconciencia y autoconocimiento. Chou et al. (2015) y Chou et al. (2016) se refieren a estas habilidades de una manera más concisa: autonomía, autorregulación, empoderamiento psicológico y autorrealización. 
En cuatro de los artículos seleccionados, se puede interpretar que las variables sociodemográficas son evaluadas como barreras para adquirir la capacidad de autodeterminación (Carter et al., 2013a, 2013b; Chou et al., 2015; Fernández et al., 2015). Carter et al. (2013a) y Carter et al. (2013b) evalúan la importancia y el desempeño o rendimiento de las habilidades de autodeterminación y la capacidad general de autodeterminación en función del sexo, nivel de discapacidad, discapacidad intelectual añadida, asistente individual, tipo de escolarización, comportamientos desafiantes, parentesco y etnia. Otros evalúan la capacidad y oportunidad de tener una conducta autodeterminada según el género, la edad, la etnia y el nivel de inteligencia (Chou et al., 2015).

Solamente uno de los trabajos analizados realiza una comparación, esta se lleva a cabo entre tres grupos, alumnado con TEA, alumnado con discapacidad intelectual y alumnado con dificultades de aprendizaje (Chou et al., 2016). Y únicamente hay un trabajo que realiza un estudio centrado en la calidad de vida, según el modelo propuesto por Schalock y Verdugo (2003), en personas con TEA según la información recogida por diferentes profesionales (García et al., 2017).

Respecto a la implementación de actividades para mejorar o desarrollar la autodeterminación, únicamente se ha obtenido un trabajo que habla de un proyecto para mejorar, entre otros aspectos, la autodeterminación de los estudiantes con TEA (Timmons et al., 2017). Este proyecto es una experiencia de servicio comunitario para el desarrollo de habilidades laborales de jóvenes con TEA en su proceso de transición a la vida adulta, con el que se ha conseguido mejorar las capacidades relacionadas con la toma de decisiones, el aumento de la autoconfianza, la creación de un sentimiento de orgullo y pertenencia al lugar y el hecho de pasar de ser receptores de servicios a ser proveedores de soluciones.

A continuación, en la Tabla 3, se desglosan los detalles de los diferentes artículos elegidos. 


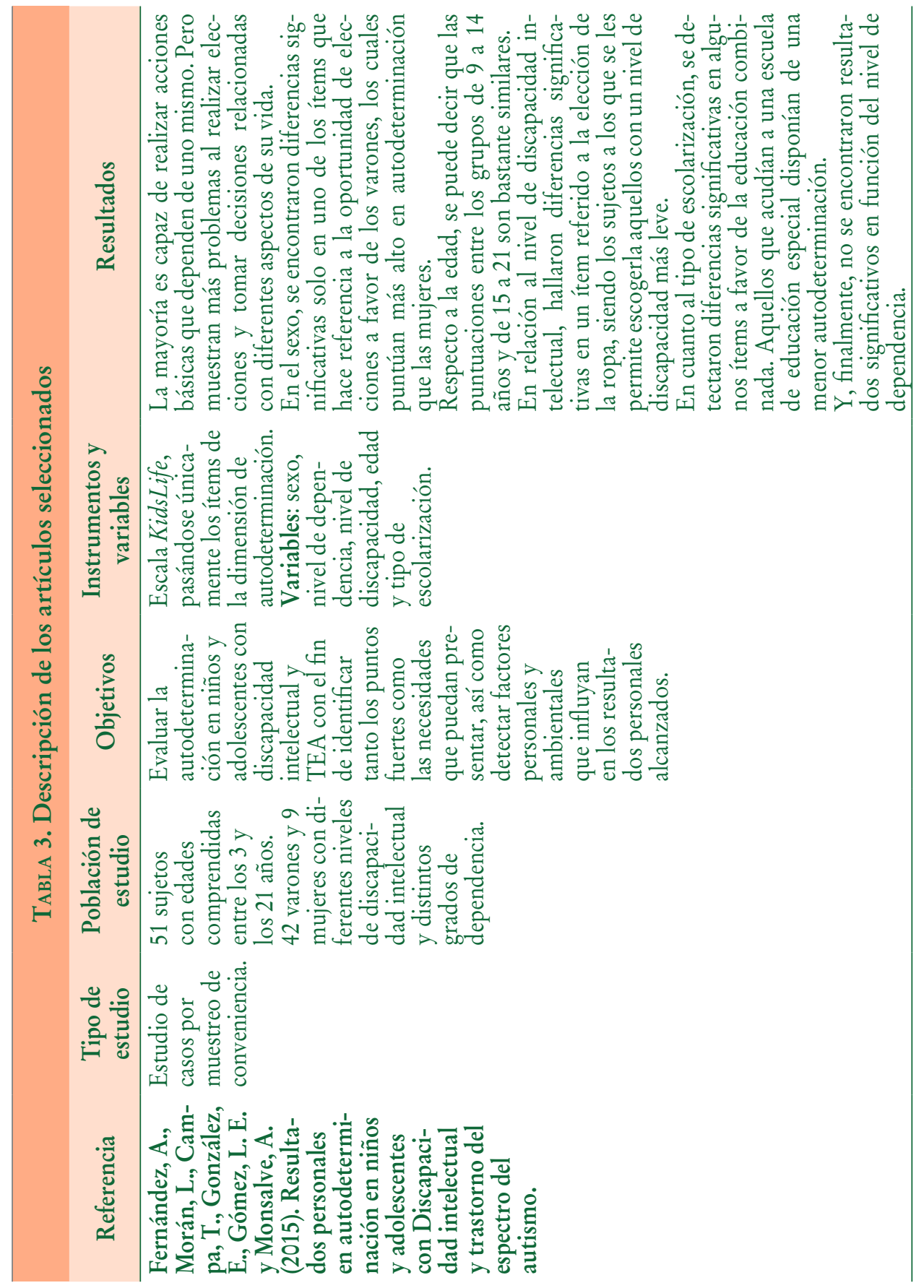

Ediciones Universidad de Salamanca / CC BY-NC-ND

Siglo Cero, vol. 52 (2), 2021, abril-junio, pp. 101-122 


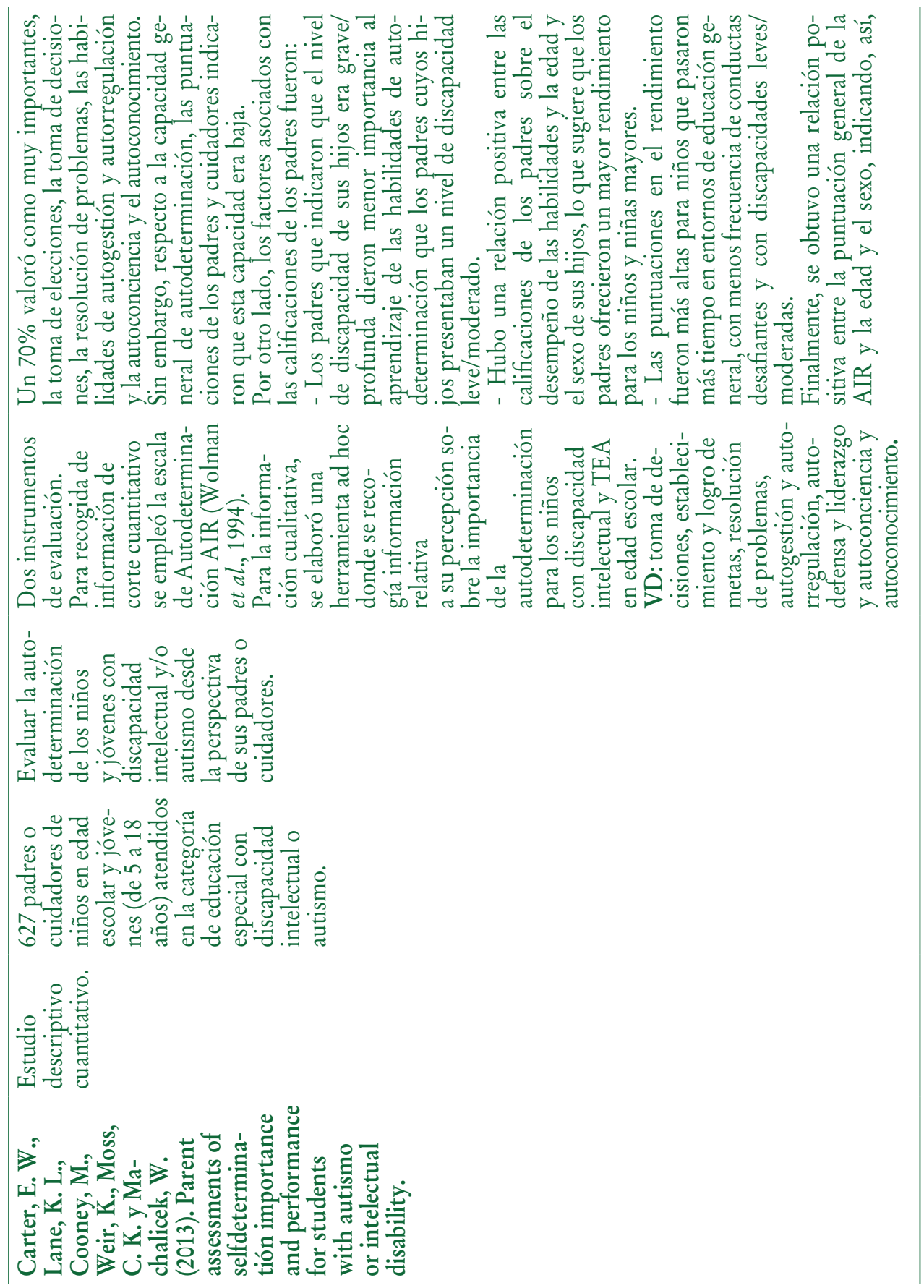

Ediciones Universidad de Salamanca / CC BY-NC-ND

Siglo Cero, vol. 52 (2), 2021, abril-junio, pp. 101-122 
AUTODETERMINACIÓN EN EL ÁMBITO EDUCATIVO

EN ADOLESCENTES CON TRASTORNO DEL ESPECTRO AUTISTA

SORAYA SÁNCHEZ CASADO, ELENA MARTÍN-PASTOR E ISABEL CALVO ÁLVAREZ

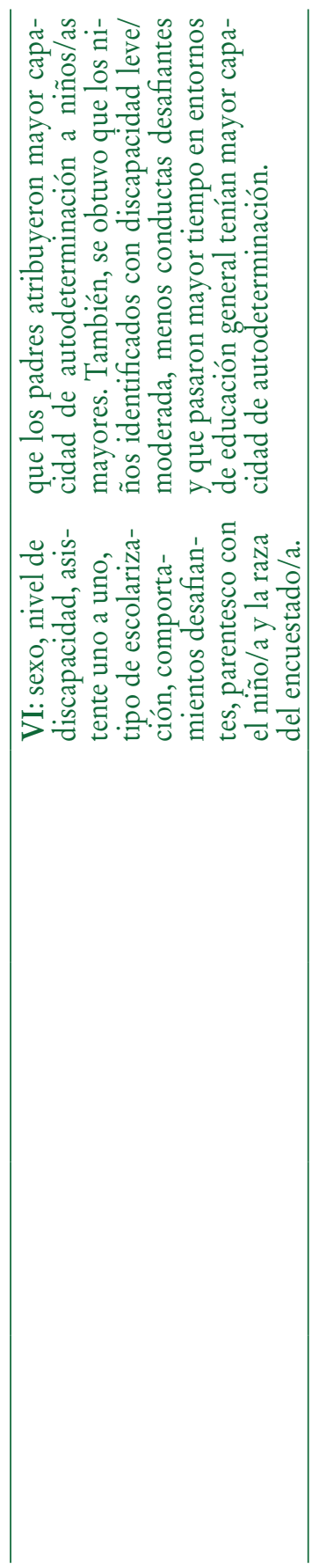

Ediciones Universidad de Salamanca / CC BY-NC-ND

Siglo Cero, vol. 52 (2), 2021, abril-junio, pp. 101-122 


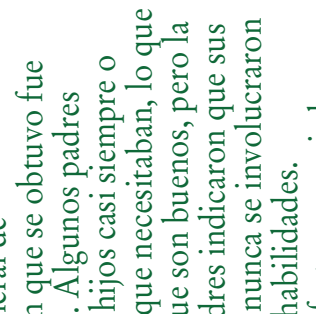

今ี

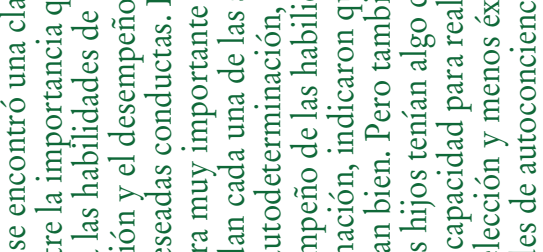

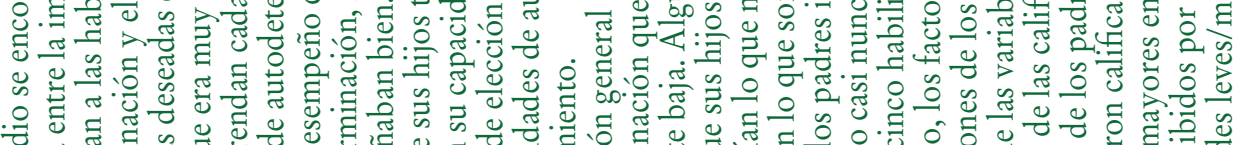

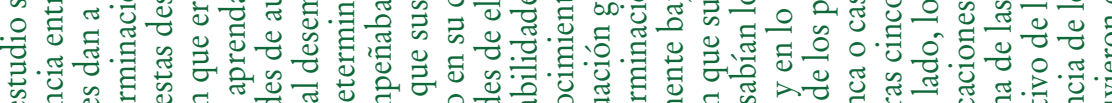

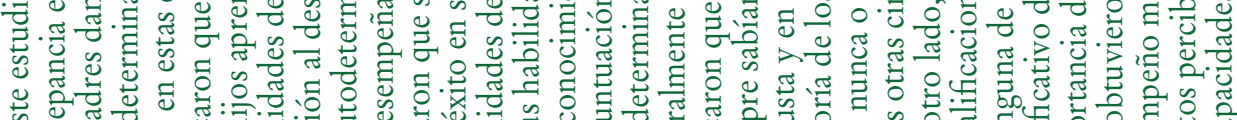

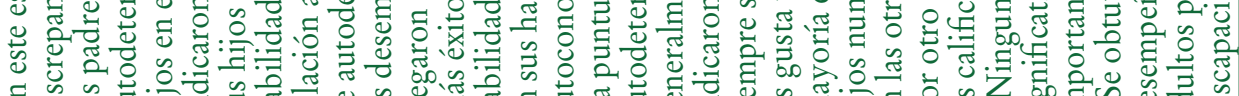

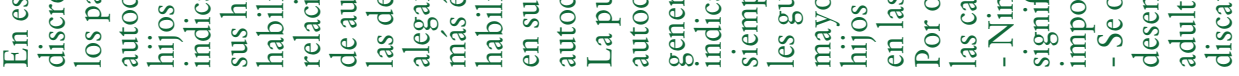

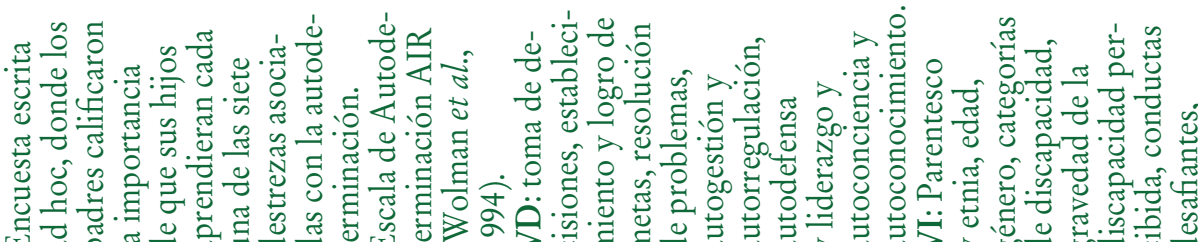

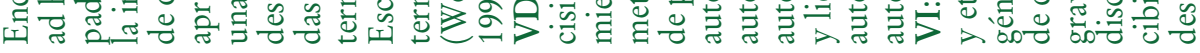

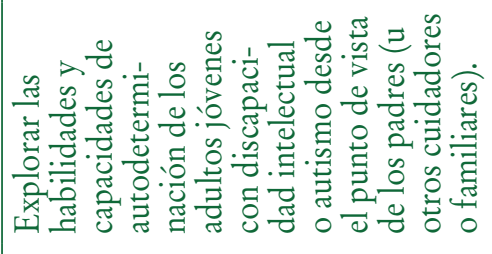

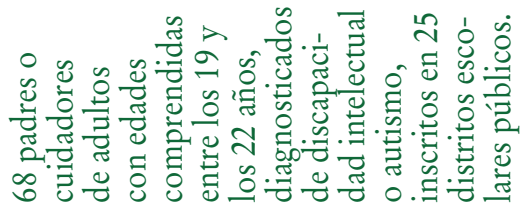

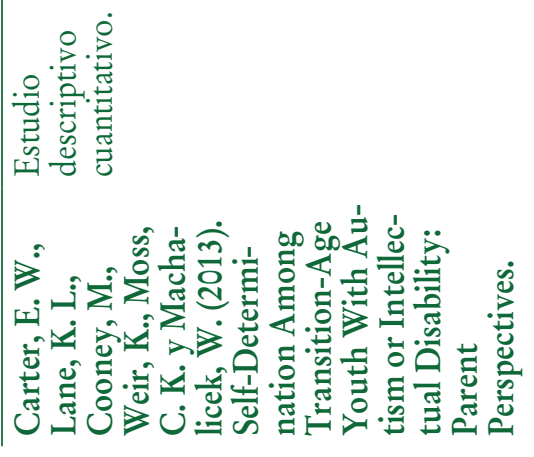

Ediciones Universidad de Salamanca / CC BY-NC-ND

Siglo Cero, vol. 52 (2), 2021, abril-junio, pp. 101-122 
AUTODETERMINACIÓN EN EL ÁMBITO EDUCATIVO

EN ADOLESCENTES CON TRASTORNO DEL ESPECTRO AUTISTA

SORAYA SÁNCHEZ CASADO, ELENA MARTÍN-PASTOR E ISABEL CALVO ÁLVAREZ

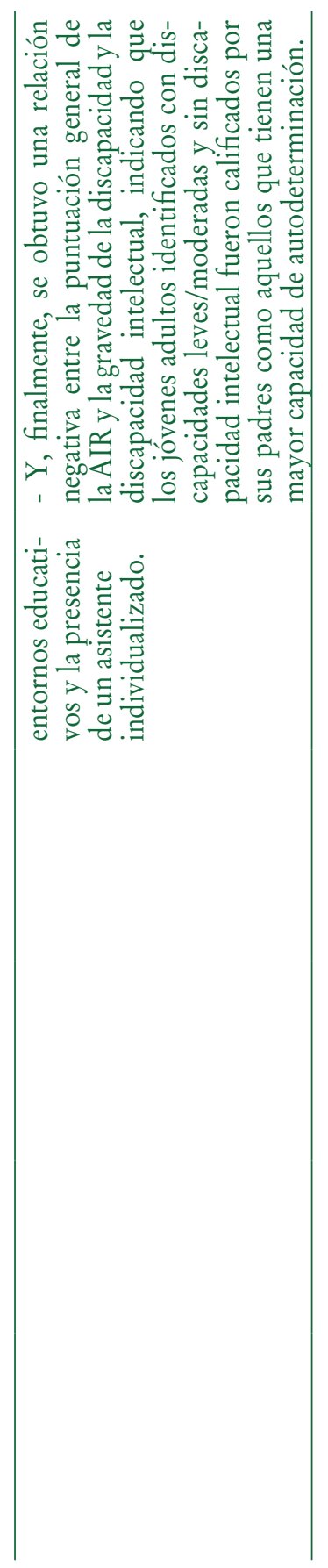

Ediciones Universidad de Salamanca / CC BY-NC-ND

Siglo Cero, vol. 52 (2), 2021, abril-junio, pp. 101-122 


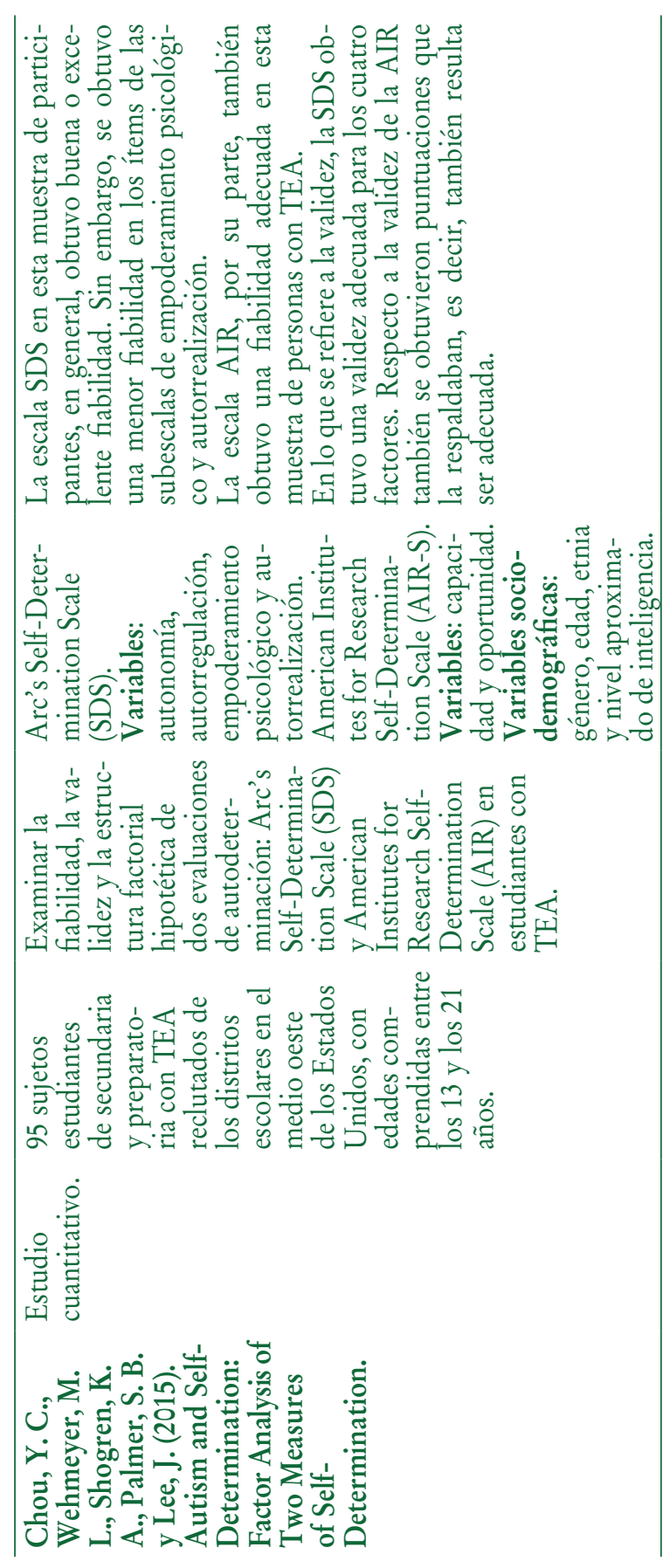

Ediciones Universidad de Salamanca / CC BY-NC-ND

Siglo Cero, vol. 52 (2), 2021, abril-junio, pp. 101-122 


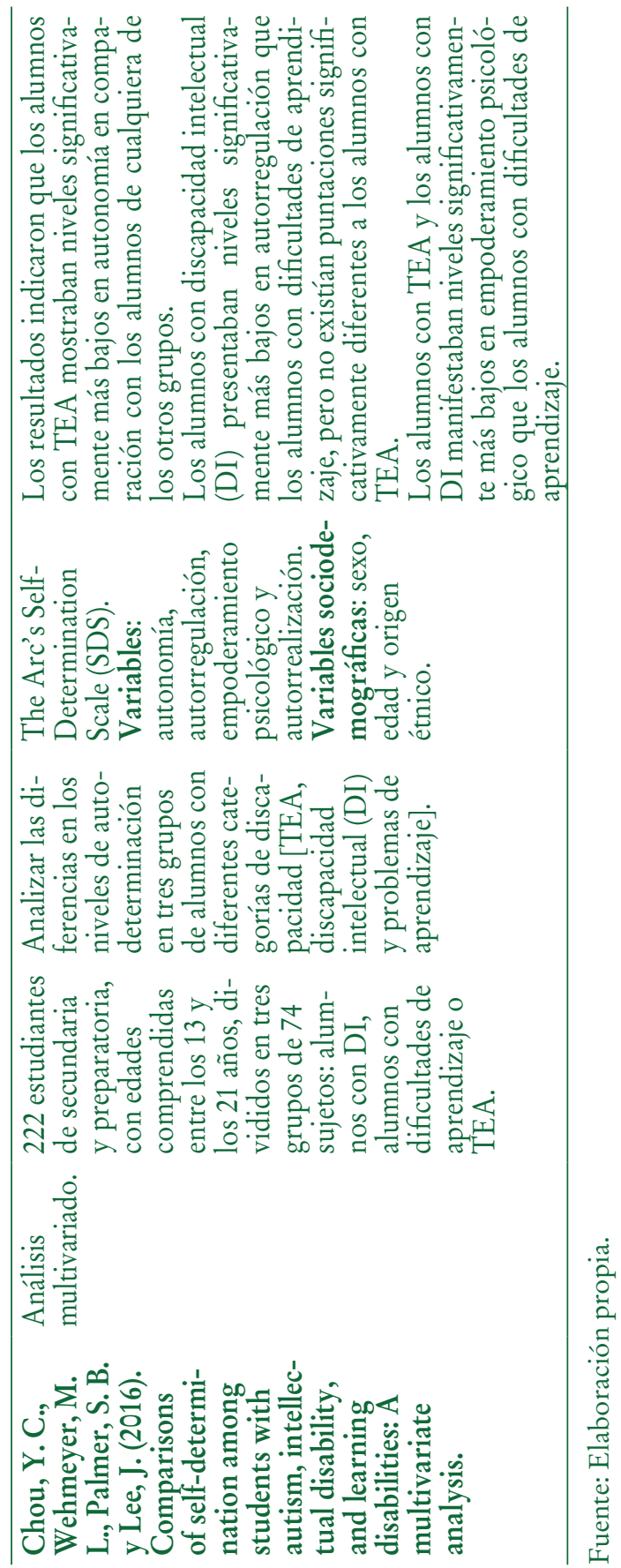

Ediciones Universidad de Salamanca / CC BY-NC-ND

Siglo Cero, vol. 52 (2), 2021, abril-junio, pp. 101-122 


\section{Discusión y conclusiones}

En la revisión realizada y los diferentes artículos analizados se ha comprobado que el estudio de la autodeterminación en adolescentes diagnosticados con trastorno del espectro autista en edad escolar es un tema que ha sido poco trabajado. Sin embargo, la autodeterminación sí que ha sido una dimensión ampliamente abordada en otros contextos y con otros colectivos con capacidades y necesidades diferentes (Nota et al., 2007; Arroyave y Freyle, 2009; Arellano y Peralta, 2013; Arellano y Peralta, 2015; Vega et al., 2017; Vicente et al., 2018; Chadwick y Fullwood, 2018; Shogren et al., 2019; Taylor et al., 2019, etc.). Esta afirmación la corroboran Chou et al. (2015) en su estudio sobre el análisis de la fiabilidad y validez de dos escalas para la evaluación de la autodeterminación en alumnado con TEA, añadiendo que se trata de un soporte preliminar para realizar futuros estudios con esta población, ya que anteriormente se ha realizado principalmente y en mayor medida con estudiantes con discapacidad intelectual y dificultades del aprendizaje. En un estudio posterior, Chou et al. (2016) afirman que, conforme al propósito de su investigación, esta es un área en la que aún existe escasa investigación.

Cuando se habla de autodeterminación en las personas con discapacidad, hablamos de derechos, de competencias, de dignidad, de acción, de ética, de responsabilidad, de autoridad y de libertad (Tamarit, 2001). Tamarit (2005) especifica que las personas con discapacidad desean adquirir mayores niveles de autodeterminación, capacidad y derecho de participación. Tras analizar la muestra de participantes de los estudios seleccionados, se ha podido ver que gran parte de los instrumentos de estas investigaciones han sido respondidos por terceras personas, padres/madres y diferentes profesionales. Estamos de acuerdo en que la información que pueden aportar es realmente importante y necesaria, pero la valoración desde la propia perspectiva de las personas con autismo resulta primordial. En estos estudios, se debería recobrar la importancia del derecho de participación de estas personas, pudiendo aportar investigaciones con resultados más significativos al tener en cuenta la valoración de ambas partes.

En relación a las barreras que las personas con autismo tienen a la hora de aumentar su nivel de autodeterminación, varios artículos recogen en sus resultados que características o variables personales como el nivel de discapacidad, el sexo, la edad, los comportamientos desafiantes, etc., influyen en los niveles de autodeterminación, siendo, por ejemplo, alumnos con discapacidades leves o moderadas los que muestran mayor conducta autodeterminada.

Las necesidades o dificultades de una persona no solo vienen determinadas por sus características personales, sino, también, por las propias barreras existentes en el entorno, siendo estas falta de recursos, de formación, actitudes negativas, etc. (Booth y Ainscow, 2015), algo que no aparece en los resultados de ninguna de las investigaciones analizadas. Sí es cierto que algunos artículos establecen el tipo de escolarización como barrera para la autodeterminación, obteniendo como resultado, en todos ellos, que el alumnado que acude a un centro de educación especial presenta menores niveles de autodeterminación. En este sentido, entendemos la educación inclusiva de 
acuerdo con Ainscow et al. (2006, citado en Sandoval et al. 2018) como "un proceso encaminado a tratar de eliminar las barreras de distinto tipo que limitan la presencia, el aprendizaje y la participación del alumnado en la vida escolar de los centros donde son escolarizados, con particular atención a aquellos más vulnerables" (p. 262).

Dando respuesta a otro de los objetivos, no se encontraron, bajo la clave de búsqueda llevada a cabo en este trabajo, programas e intervenciones desarrolladas en el ámbito educativo. El único resultado obtenido sobre programas donde se ponga en marcha el aprendizaje de la autodeterminación ha sido el estudio realizado por Timmons et al. (2017). La escasez de estudios encontrados sugiere que esta dimensión es trabajada menos de lo deseable para el logro de la inclusión de este colectivo, y/o el trabajo llevado a cabo a través de proyectos, programas y experiencias en centros educativos es de tipo regional o local sin la pertinente difusión o publicación en las bases de datos empleadas en este estudio.

Trabajos como el desarrollado por Mumbardó-Adam et al. (2017) ya pusieron de manifiesto las pocas herramientas existentes en el contexto español para el trabajo de la autodeterminación en el ámbito escolar a diferencia a lo que acontece en el contexto anglosajón.

Por último, tras el análisis de los artículos seleccionados, se observa que todos ellos arrojan resultados sobre el estado de la autodeterminación de adolescentes con autismo según sus características personales, aunque no contemplando entre sus objetivos cómo mejorar esta habilidad en estas personas. Sin considerar este dato como una limitación de los trabajos analizados, sí que se echa en falta una propuesta que oriente futuras intervenciones hacia una educación inclusiva que ofrezca los mejores apoyos al alumnado con TEA. De hecho, los artículos abordados señalan la necesidad de seguir realizando investigaciones para obtener más información sobre la capacidad autodeterminada en el alumnado con TEA. Lo anterior con la finalidad de, a la luz de los datos aportados por la realidad escolar, replantear la respuesta educativa que se proporciona a estos estudiantes con el objetivo de que se conviertan verdaderamente en los agentes causales de sus vidas y fomentar con ello un aprendizaje práctico orientado a la mejora de su calidad de vida.

\subsection{Limitaciones y prospectiva}

La principal limitación de este trabajo se concreta en la escasez de estudios encontrados que aborden el análisis de la autodeterminación en el ámbito educativo en alumnos con TEA. Además, las investigaciones analizadas estaban constituidas por muestras pequeñas, lo que dificulta la generalización de resultados.

Lo anterior pone de manifiesto la necesidad de profundizar en este campo, tanto desde el análisis del estado de la cuestión como a partir del desarrollo de estrategias y programas efectivos para promover la práctica de la autodeterminación en alumnos con autismo. De hecho, el trabajo de Timmons et al. (2017) aporta información sobre la efectividad de este tipo de programas para adquirir el aprendizaje de diferentes habilidades de la autodeterminación. O el llevado a cabo por Mumbardó-Adam et 
al. (2017), donde presentan una herramienta, desarrollada en Estados Unidos, para promover la autodeterminación -Modelo de Enseñanza y Aprendizaje de la Autodeterminación- y con posibilidades de aplicación en el contexto español que, a través de la resolución de problemas orientados a la consecución de objetivos acordes a las preferencias del alumno, se trabajan estrategias relacionadas con la autodeterminación, mejorando, indirectamente, el acceso al currículum, la participación en contextos ordinarios o la consecución de metas académicas. Con respecto a ese análisis de los niveles de autodeterminación, si bien la información proporcionada por terceras personas (familiares, profesores, cuidadores) resulta valiosa, conocer la realidad desde las propias personas implicadas -estudiantes con TEA- permitirá que el desarrollo de esas intervenciones realmente se ajuste a sus demandas y principales necesidades.

\section{Referencias bibliográficas}

American Psychiatric Association. (2018). Diagnostic and statistical manual of mental disorders (DSM-5). American Psychiatric.

Arellano, A. y Peralta, F. (2013). Calidad de vida y autodeterminación en personas con discapacidad. Valoraciones de los padres. Revista Iberoamericana de Educación, 63, 145-160.

Arellano, A. y Peralta, F. (2015). Autodeterminación personal y discapacidad intelectual: un análisis desde la perspectiva de las familias. Siglo Cero, 46(3), 7-25.

Arnaiz, P. (2019). La educación inclusiva: mejora escolar y retos para el siglo XXI. Participación Educativa. Revista del Consejo Escolar del Estado, 6(9), 41-51.

Arroyave, M. M. y Freyle, M. L. (2009). La autodeterminación en adolescentes con discapacidad intelectual. Innovar, Edición Especial en Educación, (supl. diciembre), 53-64.

BAÑA, M. (2011). Los trastornos del espectro autista y generales del desarrollo: inclusión social y calidad de vida. Ciencias Psicológicas, 5(2), 183-191.

BAÑA, M. (2015). El rol de la familia en la calidad de vida y la autodeterminación de las personas con trastorno del espectro del autismo. Ciencias Psicológicas, 9(2), 323-336.

Bonilla, M. y Chaskel, R. (2016). Trastorno del Espectro Autista. Revista Pediatría, 19-29.

Bоoтн, T. y Ainscow, M. (2015). Guía para la Educación Inclusiva. Desarrollando el aprendizaje y la participación en los centros escolares. OEI/FUHEM [Trad. cast. Воотн, T. y Ainscow, M. (2011). Index for Inclusion. Developing learning and participation in schools (3. ed. rev.). CSIE.

Carter, E. W., Lane, K. L., Cooney, M., Weir, K., Moss, C. K. y Machalicek, W. (2013a). Parent assessments of self-determination importance and performance for students with autism or intellectual disability. American Journal on Intellectual and Developmental Disabilities, 118(1), 16-31.

Carter, E. W., Lane, K. L., Cooney, M., Weir, K., Moss, C. K. y Machalicek, W. (2013b). Self-determination among transition-age youth with autism or intellectual disability: Parent perspectives. Research and Practice for Persons with Severe Disabilities, 38(3), 129-138. https://doi:10.2511/027494813809330234

Chadwick, D. D. y Fullwood, C. (2018). An online life like any other: identity, self-determination, and social networking among adults with intellectual disabilities. Cyberpsychology, Behavior and Social Networking, 21(1), 56-64. https://doi:10.1089/cyber.2016.0689

Chou, Y. C., Wehmeyer, M. L., Palmer, S. B. y Lee, J. (2016). Comparisons of self-determination among students with autism, intellectual disability, and learning disabilities: a mul- 
AUTODETERMINACIÓN EN EL ÁMBITO EDUCATIVO

EN ADOLESCENTES CON TRASTORNO DEL ESPECTRO AUTISTA

SORAYA SÁNCHEZ CASADO, ELENA MARTÍN-PASTOR E ISABEL CALVO ÁLVAREZ

tivariate analysis. Focus on Autism and other Developmental Disabilities, 32(2), 124-132. https://doi:10.1177/1088357615625059

Chou, Y.-C., Wehmeyer, M. L., Shogren, K. A., Palmer, S. B. y Lee, J. (2015). Autism and self-determination: factor analysis of two measures of self-determination. Focus on autism and other developmental disabilities, 32(3),163-175. https://doi:10.1177/1088357615611391

Echeita, G. (2017). Educación inclusiva. Sonrisas y lágrimas. Aula Abierta, 46, 17-24. https:// doi:10.17811/rifie.46.2017.17-24.

Fernández, A., Morán, L., Campa, T., González, E., Gómez, L. E. y Monsalve, A. (2015). Resultados personales en autodeterminación en niños y adolescentes con Discapacidad Intelectual y Trastorno del Espectro del Autismo. Comunicación presentada en las IX Jornadas Científicas Internacionales de Investigación sobre Personas con Discapacidad.

García, C., Sahagún, M. y Villatoro, K. (2017). Calidad de vida en personas con Trastorno del Espectro del Autismo. Orbis: Revista de Ciencias Humanas, 12(36), 65-82.

González, M. C. y Peralta, F. (2006). Autodeterminación y discapacidad: antecedentes y estado actual de un nuevo paradigma en la educación especial. Revista Española de Pedagogia, 231, 275-288.

León, M. Á. (2008). Approaching the children' families with autism. Psychosocial Intervention, $17(2), 215-230$.

Ley Orgánica 2/2006, de 3 de mayo, de Educación. BOE, 106.

Ley Orgánica 8/2013, de 9 de diciembre, para la mejora de la calidad educativa. BOE, 295.

Mumbardó-Adam, C., Vicente, E., Giné, C., Guardia-Olmos, J., Raley, S. K. y VerduGo, M. Á. (2017). Promoviendo la autodeterminación en el aula: el modelo de enseñanza y aprendizaje de la autodeterminación. Siglo Cero, 48(2), 41-59.

Nota, L., Ferrari, L., Soresi, S. y Wehmeyer, M. (2007). Self-determination, social abilities and the quality of life of people with intellectual disability. Journal of Intellectual Disability Research, 51(11), 850-865. https://doi:10.1111/j.1365-2788.2006.00939.x

Sandoval, M., Simón, C. y Márquez, C. (2018). ¿Aulas inclusivas o excluyentes?: barreras para el aprendizaje y la participación en contextos universitarios. Revista Complutense de Educación, 30(1), 261-276. https://doi:10.5209/rced.57266

Schalock, R. L. y Verdugo, M. Á. (2007). El concepto de calidad de vida en los servicios y apoyos para personas con discapacidad intelectual. Siglo Cero, 38 (4), 21-36.

Shogren, K. A., López, S. J., Wehmeyer, M. L., Little, T. D. y Pressgrove, C. L. (2006). The role of positive psychology constructs in predicting life satisfaction in adolescents with and without cognitive disabilities: an exploratory study. Journal of Positive Psychology, 1, 37- 52. https://doi:10.1080/17439760500373174

Shogren, K. A., Shaw, L. A. y Mumbardó-Adam, C. (2019). The assessment of self-determination in Spanish and American adolescents: the Self-Determination Inventory: student report. Intellectual and Developmental Disabilities, 57(4), 274-288. https://doi:10.1352/19349556-57.4.274

Shogren, K. A., Wehmeyer, M. L., Palmer, S. B., Forber-Pratt, A. J., Little, T. D. y LóPEZ, S. (2015). Causal agency theory: reconceptualizing a functional model of self-determination. Education and Training in Autism and Developmental Disabilities, 50(3), 251-263.

TAmarit, J. (2001). Propuestas para el fomento de la autodeterminación en personas con autismo y retraso mental. En M. Á. Verdugo y F. B. Jordán de Urríts (Eds.), Apoyos, autodeterminación y calidad de vida (pp. 277-292). Amarú Ediciones.

Tamarit, J. (2005). Autismo: modelos educativos para una vida de calidad. Revista de Neurología, 40(1), 181-186.

Ediciones Universidad de Salamanca / CC BY-NC-ND

Siglo Cero, vol. 52 (2), 2021, abril-junio, pp. 101-122 
Taylor, W. D., Cobigo, V. y Ouellette-Kuntz, H. (2019). A family systems perspective on supporting self-determination in young adults with intellectual and developmental disabilities. Journal of Applied Research in Intellectual Disabilities: JARID, 32(5), 1116-1128. doi:10.1111/jar.12601

The World Health Organization Quality of Life Assessment (WHOQOL). (1995). Position paper from the World Health Organization. Social Science and Medicine, 41(10), 1403-1409. https://doi:10.1016/0277-9536(95)00112-k

Timmons, J., Zalewska, A., Hall, A. C. y Fesko, S. (2017). Exploring the impact of community service on career exploration, self-determination, and social skills for transition-age youth with Autism Spectrum Disorders. Inclusion, 5(1), 16-32. https://doi:10.1352/23266988-5.1.16

UNESCO. (2017). La UNESCO avanza. La Agenda 2030 para el desarrollo sostenible. UNESCO.

Vega, V., Álvarez, I. y Jenaro, C. (2017). Autodeterminación: explorando las autopercepciones de adultos con síndrome de Down chilenos. Siglo Cero, 49(2), 89-104. https:// doi:10.14201/scero201849289104

Verdugo, M. Á., Schalock, R. L., Arias, B., Gómez, L. E. y Jordán de Urríes, B. (2013). Calidad de vida. En M. Á. Verdugo y R. L. Schalock (Coords.), Discapacidad e inclusión: manual para la docencia (pp. 443-461). Amarú.

Vicente, E., Guillén, V. M., Verdugo, M. Á. y Calvo, M. I. (2018). El rol de los factores personales y familiares en la autodeterminación de jóvenes con discapacidad intelectual. Psicología Educativa, 24(2), 75-83. https://doi:10.5093/psed2018a13

Wehmeyer, M. L. (2005). Self-determination and individuals with severe disabilities: re-examining meanings and misiterpretations. Research and Practice for Persons with Severe Disabilities, 30(3), 113-120.

Wehmeyer, M. L. (2009). Self-determination and the third generation inclusive practices. Revista de Educación, 249, 45-67.

Yianni-Coudurier, C., Darrou, C., Lenoir, P., Verrecchia, B., Assouline, B., Ledesert, B., Michelon, C., Pry, R., Aussilloux, C. y Baghdadli, A. (2008). What clinical characteristics of children with autism influence their inclusion in regular classrooms? Journal of Intellectual Disability Research, 52(10), 855-863. https://doi:10.1111/j.13652788.2008.01100.x 